\title{
Unsafe Sexual Behavior and Correlates of Risk in a Probability Sample of Men Who Have Sex With Men in the Era of Highly Active Antiretroviral Therapy
}

\author{
DEVON D. BREWER, PHD, MATTHEW R. GOLDEN, MD, MPH, AND H. HUNTER HANDSFIELD, MD
}

\begin{abstract}
Objective: To assess the levels and correlates of potential exposure to and transmission of HIV in a contemporary, community-based probability sample of men who have sex with men (MSM).

Methods: In 2003, 311 sexually active MSM participated in a random-digit dial telephone survey in Seattle neighborhoods with a high prevalence of MSM. The primary outcomes were potential exposure to and transmission of HIV, defined as unprotected anal intercourse with a man of opposite or unknown HIV status in the preceding 12 months.

Results: Fourteen percent of respondents reported being HIVpositive, $77 \%$ reported being HIV-negative, and $8 \%$ had not been tested. Of 241 HIV-negative MSM, 25 (10\%; $95 \%$ confidence interval [CI], 7-15\%) were potentially exposed to HIV; among 45 HIV-positive MSM, $14(31 \% ; 95 \%$ CI, 20-46\%) were potential HIV-transmitters. Among HIV-negative men, the strongest bivariate correlates of potential exposure to HIV were recent bacterial sexually transmitted disease (odds ratio [OR], 5.8), number of recent male sexual partners $(O R$, 1.01 per partner), recent sex at a bathhouse (OR, 9.1), and recent use of sildenafil (OR, 4.4), amyl nitrite (OR, 6.2), and methamphetamine (OR, 8.0). Among HIV-infected men, the strongest correlates of potential HIV transmission were recent use of amyl nitrite (OR, 3.1), number of recent male sex partners (OR, 1.07 per partner), and having a male spouse or domestic partner (OR, 0.3$)$.

Conclusions: Most MSM knew their HIV status and adopted safer sexual behaviors to reduce their risk of HIV acquisition or transmission. However, $10 \%$ of HIV-negative MSM and $31 \%$ of HIV-positive MSM recently engaged in behaviors that placed them at high risk for acquiring or transmitting HIV.
\end{abstract}

SINCE THE MID-1990s, SUBSTANTIAL increases in the rates of bacterial sexually transmitted diseases (STDs) have been reported in men who have sex with men (MSM) in many industrialized countries. ${ }^{1-6}$ Some data suggest that the incidence of human immunodeficiency virus (HIV) infection may also be rising in this

The authors thank JoElla Weybright, Laurie Burke, and others at Gilmore Research Group for their efforts in collecting the data and help in refining the sampling design. The authors also appreciate advice from Ron Stall and Thomas Mills about analyzing Census data on MSM.

This research was supported by a Comprehensive STD Prevention System, Syphilis Elimination grant from the Centers for Disease Control and Prevention. Robert Wood provided general support to this study and gave helpful comments on the manuscript.

Correspondence: Devon D. Brewer, PhD, Interdisciplinary Scientific Research, PO Box 15110, Seattle, WA 98115. E-mail: www.interscientific.net/ contact.html.

Received for publication May 12, 2005, and accepted August 19, 2005.
From the Department of Medicine and the Center for AIDS and STD, University of Washington, and Public Health-Seattle \& King County, Seattle, Washington

population. ${ }^{7,8}$ Recent reports indicate that risky sexual behavior in MSM - as reflected by numbers of sex partners, unprotected anal intercourse (UAI), and other measures - may have been increasing during this time as well. $3,4,9,10$ Most investigators have attributed these trends directly or indirectly to improved treatment of HIV infection and the acquired immunodeficiency syndrome (AIDS) with highly active antiretroviral therapy (HAART). However, all recent data on sexual behavior in MSM have come from convenience samples, typically MSM with reported STDs, attendees of public STD clinics, persons receiving clinical or prevention services, or MSM contacted through outreach. Except for general population surveys that included relatively few MSM and limited assessment of MSM sexual behavior, ${ }^{11,12}$ the only reported population-based surveys of sexual behavior in MSM were performed before the development and widespread use of HAART. Therefore, we undertook this study to estimate the frequency and correlates of sexual behaviors conducive to acquisition or transmission of HIV in a probability sample of MSM.

\section{Methods}

We conducted a random-digit dial (RDD) telephone household survey of MSM in King County, Washington (population 1.7 million), which encompasses the city of Seattle (population 563,000). The sampling universe included all households with telephones in zip (postal) codes with high estimated concentrations of MSM. The estimated geographic distribution of MSM was based on: 1) the proportion of households with unmarried males living with male partners in the 2000 U.S. Census (www.census. gov); 2) MSM seen in the Public Health-Seattle \& King County (PHSKC) STD Clinic in 2000 and 2001 and in the public health HIV testing and counseling program from 1998 through 2001; 3) newly diagnosed HIV cases in MSM reported in King County from 1999 through 2001; and 4) an anonymous probability sample of patrons of a local bathhouse in 2001 (sources 2-4 are unpublished PHSKC data). All four data sources revealed similar geographic distributions of MSM in King County. We limited the telephone survey to three contiguous zip codes in central Seattle that had the highest estimated prevalences of MSM and included $21 \%$ to $37 \%$ of the MSM in these data sources. To determine the 
telephone prefixes (exchanges) for the sampling frame, we assessed data from Genesys Sampling Systems (Marketing Systems Group, Fort Washington, PA) on the telephone prefixes and zip codes of households with listed landline telephone numbers. We selected 13 prefixes that covered $97 \%$ of the listed numbers, and presumably a similar percentage of unlisted numbers, in the three target zip codes as of May 2002. These prefixes overlapped with, but were not exclusive to, the target zip codes. This sampling frame did not include numbers for cellular phones.

From February 3 to May 18, 2003, Gilmore Research Group (Seattle, WA) used RDD to sample households with the selected telephone prefixes and conducted computer-assisted telephone interviews. Calling occurred during each day of the week and at different times (morning, afternoon, and evening). Interviewers called each sampled number at least eight times on different days and at different times before stopping contact attempts. When a household was contacted, interviewers first elicited the household's zip code and then proceeded with screening if it was in a targeted zip code. If more than one man $\geq 18$ years old lived in a contacted household, one man was randomly selected for participation in the survey. After introducing the nature of the survey, the interviewer asked whether the respondent had ever had sex with another man since age 14; those responding affirmatively were eligible to participate. Respondents participated anonymously. Survey questions covered respondents' STD/HIV testing and history, substance use, sexual behavior, related attitudes, and demographics. The number of possible postscreening questions ranged from 11 to 121 as a result of branching and skip patterns. The project was determined by the University of Washington Human Subjects Review Committee to be exempt from a requirement for informed consent.

Interviewers attempted a total of 17,105 calls and screened 5077 households. The response rate, defined as the number of completed interviews divided by the estimated number of all telephone numbers dialed that belonged to households with at least one MSM, was $46 \%$ (AAPOR RR3). ${ }^{13}$ This rate is based on the assumption that telephone numbers not contacted or screened for eligibility had a similar proportion of eligible households as those contacted and screened. Eight percent of households screened in the target zip codes had at least one MSM. Of 412 initially identified MSM, $400(97 \%)$ completed the survey. Interviewers made 3.8 call attempts, on average, to these 400 respondents' households (median, 3; range, 1-18). Interviews lasted a mean of 13 minutes (range, 3-28 minutes). Of the 400 respondents, 311 (78\%) reported sex with another man in the preceding 12 months; these 311 men comprised the sample for analysis.

The primary outcomes were potential exposure to and transmission of HIV. Respondents who described themselves as HIVnegative were classified as potentially exposed if they reported either insertive or receptive unprotected anal intercourse (UAI) in the preceding 12 months with at least one man whom the respondent believed to be HIV-positive or whose HIV status was unknown. Similarly, HIV-positive respondents were classified as potential transmitters if they reported UAI in the preceding year with a man who was HIV-negative or of unknown HIV status.

\section{Statistical Analysis}

We performed bivariate logistic regression analyses with potential exposure to and transmission of HIV as the dependent variables. We calculated the $95 \%$ confidence intervals (CIs) for each odds ratio (OR). We also computed a multivariate logistic regression model with potential exposure as the dependent variable and those independent variables that had statistically significant $(P<$ $0.05)$ bivariate ORs.

\section{Results}

Of the 311 respondents, 45 (14\%) reported being HIV-positive and $241(77 \%)$ reported testing HIV-negative at their most recent test; $73 \%$ of the HIV-negative men had been tested within the previous 12 months. The remaining 25 respondents $(8 \%)$ denied having been tested or had tested but did not know their HIV status. Of the 241 HIV-negative men, 25 (10\%; 95\% CI, 7-15\%) were potentially exposed to HIV, and $14(31 \%$; 95\% CI, 20-46\%) of the $45 \mathrm{HIV}$-infected men were potential HIV-transmitters.

Table 1 shows the correlates of potential exposure in HIVnegative respondents. Potentially exposed men were somewhat younger than those who were not potentially exposed, but the two groups were otherwise demographically similar. Potentially exposed MSM were more likely to have had a diagnosed bacterial STD (chlamydia, gonorrhea, or syphilis) in the past 12 months (OR, 5.75) and to have used sildenafil (Viagra) (OR, 4.35), amyl nitrite (OR, 6.20), and methamphetamine (OR, 8.04) in the preceding 6 months. Binge alcohol use ( $>5$ drinks during 1 day) in the last 30 days was unrelated to potential exposure. Potentially exposed MSM had approximately four times as many male sex partners in the preceding 12 months as those not exposed, on average. The 25 potentially exposed MSM reported 871 male sex partners and 324 male anal sex partners in the last 12 months; these represent $30 \%$ of all male sexual partnerships and $42 \%$ of all male anal sex partnerships, respectively, reported by the 240 HIVnegative MSM for whom data were available. The strongest correlate of potential exposure to HIV was having sex at a bathhouse in the last 12 months (OR, 9.14). Sex with female partners and having a male spouse or domestic partner were not meaningfully associated with potential exposure. Sex at a bathhouse (OR, 7.07), sildenafil use (OR, 5.47), and younger age (OR, 0.89 per year) remained strong and significant correlates of potential exposure in the multivariate analysis. Other analyses indicated that the 25 MSM who did not know their HIV status were behaviorally and demographically similar to HIV-negative men who were not potentially exposed (data not shown).

Table 2 shows the correlates of potential HIV transmission among the smaller number of HIV-positive MSM. In general, few variables were meaningfully or significantly associated with potential transmission. Potential transmitters were substantially, albeit nonsignificantly, more likely to have used amyl nitrite in the preceding 6 months (OR, 3.13). Potential transmitters also had three times as many male sex partners in the last 12 months as nontransmitters on average. The 14 men classified as potential transmitters reported 326 male sex partners and 174 male anal sex partners in the prior 12 months; these represent $58 \%$ of all male sexual partnerships and $57 \%$ of all male anal sexual partnerships, respectively, reported by the $44 \mathrm{HIV}$-positive MSM for whom data were available. HAART use was not substantially related to potential transmission $(\mathrm{OR}, 0.64)$ or UAI regardless of partner status (OR, 0.65).

Respondents reported higher levels of UAI with partners of concordant HIV status. Ninety-two (38\%) of the $241 \mathrm{HIV}$-negative men reported UAI with HIV-negative partners and 22 (49\%) of the 45 HIV-positive men reported UAI with HIV-positive partners.

\section{Discussion}

Our results have both encouraging and unsettling implications for HIV prevention in MSM. Most HIV-negative MSM in our sample had been tested for HIV in the preceding year. Despite high and continued rising rates of STD in Seattle MSM since 1997 (Public Health-Seattle \& King County, unpublished data), ${ }^{2} 90 \%$ of HIV-negative respondents in our probability sample denied engag- 
TABLE 1. Correlates of Potential Exposure in HIV-Negative Men Who Have Sex With Men, Seattle, 2003*

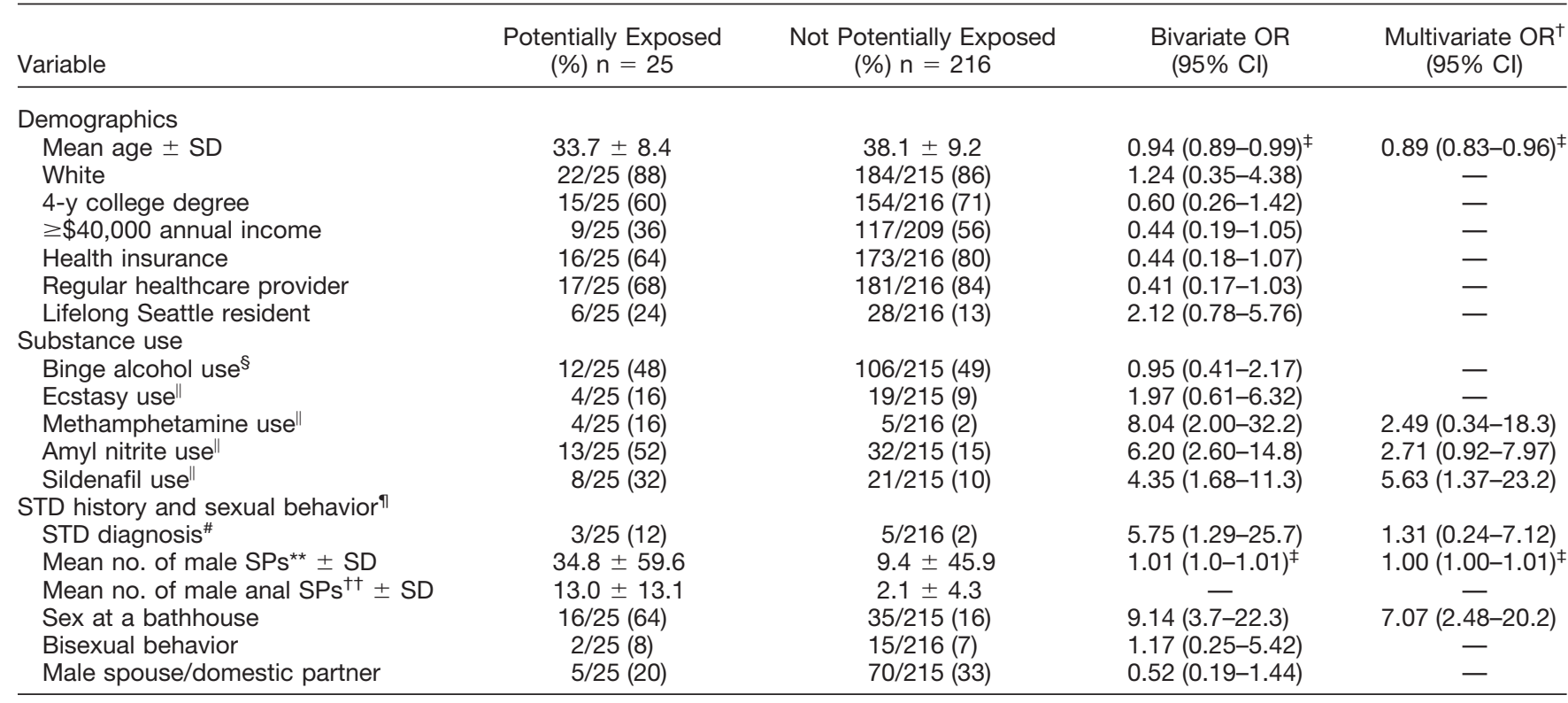

${ }^{*}$ Potential exposure refers to unprotected anal intercourse with a male sex partner who is HIV-positive or of unknown HIV status.

${ }^{t} \mathrm{n}=238$.

${ }^{\ddagger}$ OR per additional unit (year of age, male sex partner).

\$Past $30 \mathrm{~d}$.

"Past 6 mo.

"Current male spouse/domestic partner; other STD history and sexual behavior variables refer to last 12 mo.

\#Diagnosis of chlamydia, gonorrhea, and/or syphilis.

**Medians $=15$ for potentially exposed and 2 for not potentially exposed.

${ }^{+\dagger}$ Medians $=9$ for potentially exposed and 1 for not potentially exposed; no OR computed because predictor variable confounded with potential exposure.

$\mathrm{Cl}$ indicates confidence interval; OR = odds ratio; SD = standard deviation; STD = sexually transmitted disease; SPs = sex partners.

ing in behaviors in the year preceding the survey that would place them at high risk for acquiring HIV. These results indicate that it is normative for HIV-uninfected MSM to know their HIV status and take measures that reduce their risk of becoming infected. At the same time, it is disconcerting that in the preceding year, almost one third of HIV-infected MSM had engaged in behavior that carried a high risk of transmission to their sex partners. On the other hand, two thirds of the potential transmitters in our sample were taking HAART, which may reduce infectivity, ${ }^{14-17}$ although such reductions might be somewhat offset by increased infectivity in the presence of urethritis. ${ }^{18,19}$ Some men undoubtedly have varied behavior patterns over time, so the proportions of MSM at risk for HIV acquisition or transmission for intervals longer than 1 year undoubtedly exceed our estimates.

The level of potential exposure in our probability sample is less than that observed in convenience samples of MSM drawn from gyms (health clubs) and organizations providing clinical and prevention services to MSM and community outreach in the HAART era. ${ }^{20-22}$ Other studies of MSM in both the HAART and preHAART eras indicated lower levels of potential exposure and transmission ${ }^{23-25}$ but were based on less comprehensive measures of risk that are not comparable to those we used.

The potentially exposed respondents were a definable subset of HIV-negative MSM. The strongest correlates of potential exposure were young age, recent diagnosis of bacterial STD, large numbers of male sex partners, having sex at a bathhouse, and recent use of sildenafil, amyl nitrite, and methamphetamine. However, potential HIV-transmitters were less easily characterized among HIV-posi- tive MSM. Young age, recent use of amyl nitrite, and many recent male sex partners were moderately associated with potential transmission behavior.

The strongest correlates of potential exposure to HIV in our sample are also the factors most correlated with incident HIV infection in a large multisite cohort study of MSM in the United States conducted in 1998-199926 and prevalent self-reported HIV in our probability sample as well as in MSM patients with STDs and HIV testing clients in Seattle. ${ }^{21}$ Furthermore, potential exposure to HIV, defined as in the current study, was the best predictor of new HIV diagnosis in MSM patients with STDs in Seattle. ${ }^{27}$ These converging results demonstrate the value of potential exposure as an indicator of HIV risk.

Establishing a causal link between substance use and high-risk sex is difficult, but other evidence suggests that amphetamines may promote high-risk sexual behavior, including: a substantial and consistent association between amphetamine use and high-risk sexual behavior and HIV acquisition, ${ }^{26,28-33}$ widespread use of methamphetamines among MSM for the expressed purpose of enhancing sex, ${ }^{34}$ increased frequency of sexual intercourse in laboratory animals experimentally administered amphetamines. ${ }^{35,36}$ and decreased methamphetamine use and high-risk sex in response to drug abuse treatment. ${ }^{37}$ Such results highlight the need for studying event-level associations between the use of particular drugs and high-risk sex. ${ }^{38-40}$

Several methodological issues should be considered when interpreting our results. First, the measures of potential exposure and transmission are not perfect assays of HIV risk. Apart from the 
TABLE 2. Correlates of Potential HIV Transmission in HIV-Positive Men Who Have Sex With Men, Seattle, 2003*

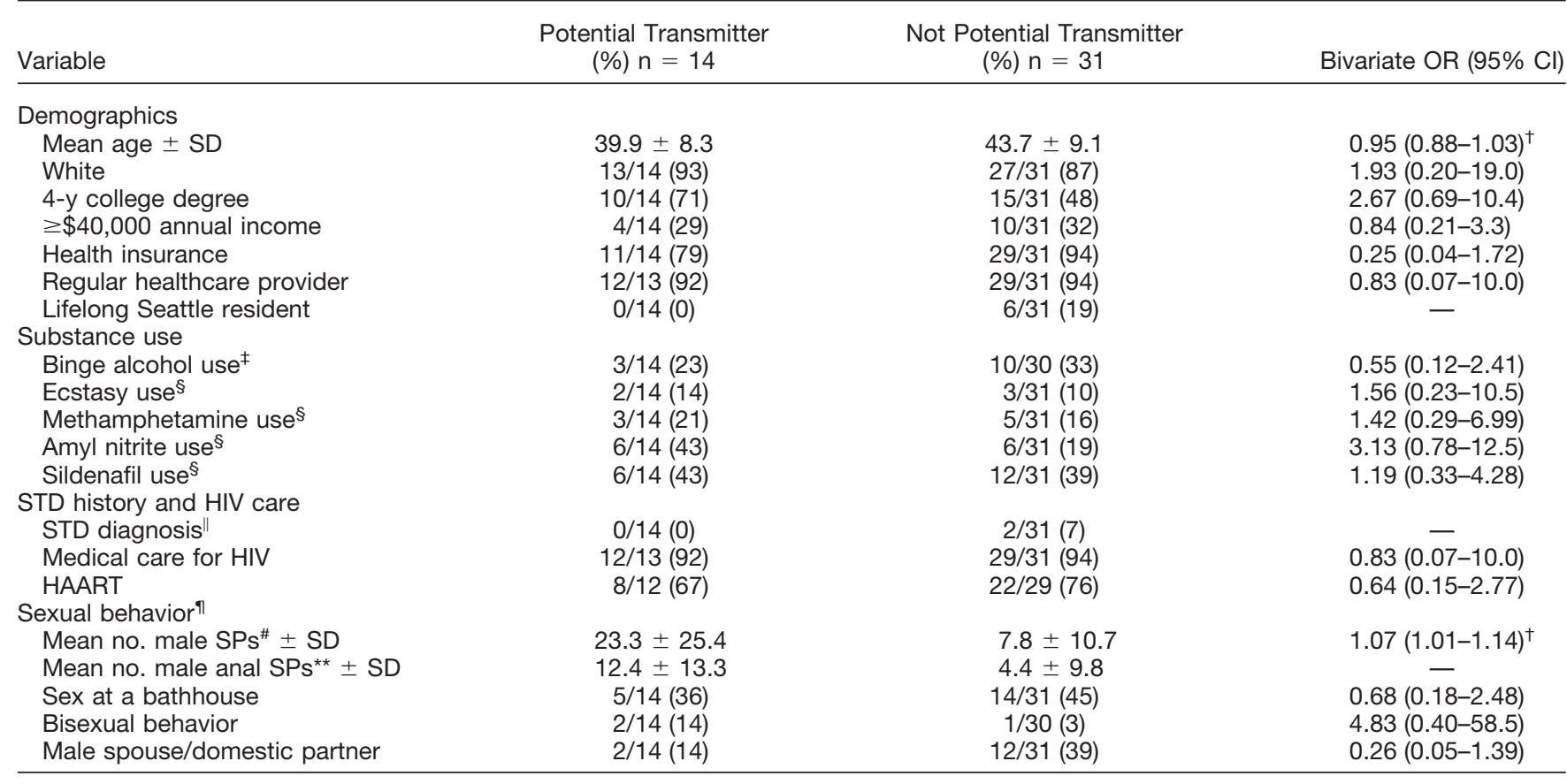

*Potential transmission refers to unprotected anal intercourse with a male sex partner who is HIV-negative or of unknown HIV status.

${ }^{\dagger}$ OR per additional unit (year of age, male sex partner).

₹Past $30 \mathrm{~d}$.

§Past 6 mo.

"Diagnosis of chlamydia, gonorrhea, or syphilis in last 12 mo.

"Current male spouse/domestic partner; other sexual behavior variables refer to last $12 \mathrm{mo}$.

\#Medians $=14$ for potential transmitters and 4 for not potential transmitters.

${ }^{*}$ Medians $=6$ for potential transmitters and 2 for not potential transmitters; no OR computed because predictor variable confounded with potential transmission.

OR indicates odds ratio; $\mathrm{Cl}=$ confidence interval; $\mathrm{SD}=$ standard deviation; STD = sexually transmitted disease; HAART = highly active antiretroviral therapy; SPs $=$ sex partners.

usual possible sources of error and bias in self-reports, respondents' partners may not have accurately reported their HIV status, and sexual practices other than UAI involve some risk.

With respect to sampling, the exclusion of cellular telephone numbers from our sampling frame may have introduced some small bias. In 2003, the year our survey was conducted, approximately $4 \%$ of U.S. households had cellular phone service only, and the characteristics of our sample included factors both positively and negatively associated with such service (Blumberg, Luke, and Cynamon, unpublished data; Tucker and Brick, unpublished data). In addition, the men in our sample, who resided in neighborhoods with relatively high densities of MSM, may not be behaviorally representative of MSM in the whole Seattle metropolitan area or of MSM nationally. However, geographically concentrated urban populations of MSM probably are common across the United States, and our sample resembles probability samples of MSM in other cities in the pre-HAART era on several demographic characteristics. $^{41-43}$ Furthermore, response rates in RDD telephone surveys tend to be lower than those obtained in household surveys conducted face-to-face. Nonetheless, the $46 \%$ response rate we observed approximates that for other RDD telephone surveys such as the national 2003 Survey of Consumer Attitudes (48\%) (4) $^{4}$ and the 2002 Behavioral Risk Factor Surveillance Survey in Washington state $(54 \%)$, which was carried out by the same telephone survey firm that collected our data. ${ }^{45}$ Catania et $\mathrm{al}^{41}$ did not report a response rate for their 1997 RDD survey of MSM in San
Francisco, Los Angeles, Chicago, and New York City, but their completion (cooperation) ${ }^{13}$ rate $(78 \%)$ was substantially lower than ours (97\%). Finally, the small number of HIV-infected MSM in our sample produced large CIs around the generally modest associations we observed for potential transmission. Despite these sampling limitations, RDD procedures were the only practical and cost-effective means to sample MSM probabilistically, and the resulting sample surely is more representative than the convenience samples of MSM reported in the HAART era.

Our findings suggest several potential avenues for advancing HIV prevention in MSM. In clinical settings, routinely asking MSM integrated questions about their condom use with and HIV status of their anal sex partners can improve risk assessment and sharpen the focus for client-centered risk-reduction counseling. Behavioral interventions might be most productively focused on MSM STD cases, bathhouse patrons, and users of methamphetamines, amyl nitrite, and sildenafil, because these men have the greatest risk of exposure. Many such men are readily identifiable and accessible through STD clinics, routine STD case-reporting to health departments, and outreach. Similarly, programs designed to curb use of methamphetamine, amyl nitrite, and sildenafil in MSM might help reduce HIV acquisition. Structural and regulatory interventions concerning bathhouses and similar venues might also inhibit HIV transmission in MSM. Finally, community-level riskreduction campaigns and individual counseling approaches that invoke observed norms of low-risk sexual behavior may also hold 
promise. Rigorous evaluations of each of these strategies are needed to determine their preventive value.

\section{References}

1. Johansen JD, Smith E. Gonorrhoea in Denmark: High incidence among HIV-infected men who have sex with men. Acta Derm Venereol 2002; 82:365-368.

2. Centers for Disease Control and Prevention (CDC). Resurgent bacterial sexually transmitted disease among men who have sex with men—King County, Washington, 1997-1999. MMWR Morb Mortal Wkly Rep 1999; 48:773-777.

3. Rietmeijer CA, Patnaik JL, Judson FN, et al. Increases in gonorrhea and sexual risk behaviors among men who have sex with men: A 12-year trend analysis at the Denver Metro Health Clinic. Sex Transm Dis 2003; 30:562-567.

4. Chen SY, Gibson S, Katz MH, et al. Continuing increases in sexual risk behavior and sexually transmitted diseases among men who have sex with men: San Francisco, Calif, 1999-2001, USA. Am J Public Health 2002; 92:1387-1388.

5. Wolitski RJ, Valdiserri RO, Denning PH, et al. Are we headed for a resurgence of the HIV epidemic among men who have sex with men? Am J Public Health 2001; 91:883-888.

6. Stolte IG, Dukers NH, de Wit JB, et al. Increase in sexually transmitted infections among homosexual men in Amsterdam in relation to HAART. Sex Transm Infect 2001; 77:184-186.

7. Calzavara L, Burchell AN, Major C, et al. Increases in HIV incidence among men who have sex with men undergoing repeat diagnostic HIV testing in Ontario, Canada. AIDS 2002; 16:16551661.

8. Katz MH, Schwarcz SK, Kellogg TA, et al. Impact of highly active antiretroviral treatment on HIV seroincidence among men who have sex with men: San Francisco. Am J Public Health 2002; 92:388-394.

9. Chen SY, Gibson S, Weide D, et al. Unprotected anal intercourse between potentially HIV-serodiscordant men who have sex with men, San Francisco. J Acquir Immune Defic Syndr 2003; 33:166170.

10. Dodds JP, Mercey DE, Parry JV, et al. Increasing risk behaviour and high levels of undiagnosed HIV infection in a community sample of homosexual men. Sex Transm Infect 2004; 80:236-240.

11. Lau JTF, Kim JH, Lau M, et al. HIV related behaviours and attitudes among Chinese men who have sex with men in Hong Kong: A population based study. Sex Transm Infect 2004; 80:459-465.

12. Mercer CH, Fenton KA, Copas AJ, et al. Increasing prevalence of male homosexual partnerships and practices in Britain 1990-2000: Evidence from national probability surveys. AIDS 2004; 18:1453-1458.

13. American Association for Public Opinion Research (AAPOR). Standard Definitions: Final Dispositions of Case Codes and Outcome Rates for Surveys. Ann Arbor, MI: AAPOR, 2000.

14. Barroso PF, Schechter M, Gupta P, et al. Effect of antiretroviral therapy on HIV shedding in semen. Ann Intern Med 2000; 133:280284.

15. Lampinen TM, Critchlow CW, Kuypers JM, et al. Association of antiretroviral therapy with detection of HIV-1 RNA and DNA in the anorectal mucosa of homosexual men. AIDS 2000; 14: F69-75.

16. Vernazza PL, Gilliam BL, Flepp M, et al. Effect of antiviral treatment on the shedding of HIV-1 in semen. AIDS 1997; 11:1249-1254.

17. Zuckerman RA, Whittington WL, Celum CL, et al. Higher concentration of HIV RNA in rectal mucosa secretions than in blood and seminal plasma, among men who have sex with men, independent of antiretroviral therapy. J Infect Dis 2004; 190:156-161.

18. Winter AJ, Taylor S, Workman J, et al. Asymptomatic urethritis and detection of HIV-1 RNA in seminal plasma. Sex Transm Infect 1999; 75:261-263.

19. Sadiq ST, Taylor S, Kaye S, et al. The effects of antiretroviral therapy on HIV-1 RNA loads in seminal plasma in HIV-positive patients with and without urethritis. AIDS 2002; 16:219-225.

20. Colfax G, Vittinghoff E, Husnik MJ, et al. Substance use and sexual risk: A participant- and episode-level analysis among a cohort of men who have sex with men. Am J Epidemiol 2004; 159:10021012.

21. Brewer DD, Golden MR, Krekeler B, et al. Comparison of men who have sex with men (MSM) in a community probability sample with MSM in clinical samples. Abstract P146, presented at the National STD Prevention Conference, Philadelphia, March 2004.

22. Elford J, Bolding G, Davis M, et al. Trends in sexual behaviour among London homosexual men 1998-2003: Implications for HIV prevention and sexual health promotion. Sex Transm Infect 2004; 80:451454

23. Paul JP, Catania J, Pollack L, et al. Understanding childhood sexual abuse as a predictor of sexual risk-taking among men who have sex with men: The Urban Men's Health Study. Child Abuse Negl 2001; 25:557-584.

24. Weinhardt L, Kelly J, Brondino M, et al. HIV Transmission risk behavior among men and women living with HIV in 4 cities in the United States. J Acquir Immune Defic Syndr 2004; 36:1057-1066.

25. Centers for Disease Control and Prevention. High-risk sexual behavior by HIV-positive men who have sex with men-16 sites, United States, 2000-2002. MMWR Morb Mortal Wkly Rep 2004; 53:891894.

26. Ackers M, Greenberg A, Lin C, et al. High HIV incidence among men who have sex with men participating in an HIV vaccine efficacy trial, United States, 1998-2002. Abstract 857, presented at the 11th Conference on Retroviruses and Opportunistic Infections, San Francisco, February 8-11, 2004.

27. Golden MR, Brewer DD, Kurth A, et al. Importance of sex partner HIV status in HIV risk assessment among men who have sex with men. J Acquir Immune Defic Syndr 2004; 36:734-742.

28. Burcham JL, Tindall B, Marmor M, et al. Incidence and risk factors for human immunodeficiency virus seroconversion in a cohort of Sydney homosexual men. Med J Aust 1989; 150:634-639.

29. Page-Shafer K, Veugelers PJ, Moss AR, et al. Sexual risk behavior and risk factors for HIV-1 seroconversion in homosexual men participating in the Tricontinental Seroconverter Study, 1982-1994. Am J Epidemiol 1997; 146:531-542.

30. Chesney MA, Barrett DC, Stall R. Histories of substance use and risk behavior: Precursors to HIV seroconversion in homosexual men. Am J Public Health 1998; 88:113-116.

31. Whittington WL, Collis T, Dithmer-Schreck D, et al. Sexually transmitted diseases and human immunodeficiency virus-discordant partnerships among men who have sex with men. Clin Infect Dis 2002; 35:1010-1017.

32. Harris NV, Thiede H, McGough JP, et al. Risk factors for HIV infection among injection drug users: Results of blinded surveys in drug treatment centers, King County, Washington 1988-1991. J Acquir Immune Defic Syndr 1993; 6:1275-1282.

33. Colfax GN. The epidemiology of substance use and sexual risk behavior among men who have sex with men: Implications for HIV prevention interventions. Paper presented at the 12th Conference on Retroviruses and Opportunistic Infections, Boston, February 22-25, 2005.

34. Reback CJ. The Social Construction of a Gay Drug: Methamphetamine Use Among Gay and Bisexual Males in Los Angeles. Los Angeles: AIDS Coordinators Office, 1997.

35. Bignami G. Pharmacologic influences on mating behavior in the male rat. Effects of d-amphetamine, LSD-25, strychnine, nicotine and various anticholinergic agents. Psychopharmacologia 1966; 10:44-58.

36. Fiorino DF, Phillips AG. Facilitation of sexual behavior in male rats following d-amphetamine-induced behavioral sensitization. Psychopharmacology (Berl) 1999; 142:200-208.

37. Shoptaw S, Reback CJ, Peck JA, et al. Behavioral treatment approaches for methamphetamine dependence and HIV-related sexual risk behaviors among urban gay and bisexual men. Drug Alcohol Depend 2005; 78:125-134.

38. Leigh BC. Alcohol and condom use: A meta-analysis of event-level studies. Sex Transm Dis 2002; 29:476-482.

39. Vanable PA, McKirnan DJ, Buchbinder SP, et al. Alcohol use and high-risk sexual behavior among men who have sex with men: The effects of consumption level and partner type. Health Psychol 2004; 23:525-532. 
40. Weinhardt LS, Carey MP. Does alcohol lead to sexual risk behavior? Findings from event-level research. Annu Rev Sex Res 2000; 11: $125-157$.

41. Catania JA, Osmond D, Stall RD, et al. The continuing HIV epidemic among men who have sex with men. Am J Public Health 2001; 91:907-914.

42. Webster RD, Darrow WW, Paul JP, et al. HIV infection and associated risks among young men who have sex with men in a Florida resort community. J Acquir Immune Defic Syndr 2003; 33:223-231.
43. Jinich S, Paul JP, Stall R, et al. Childhood sexual abuse and HIV risk-taking behavior among gay and bisexual men. AIDS Behav 1998; 2:41-51.

44. Curtin R, Presser S, Singer E. Changes in telephone survey nonresponse over the past quarter century. Public Opin Q 2005; 69:87-98.

45. Centers for Disease Control and Prevention (CDC). 2002 BRFSS Summary Data Quality Report. Available at: http://www.cdc.gov/ brfss/technical_infodata/2002QualityReport.htm. Accessed September 9, 2004. 